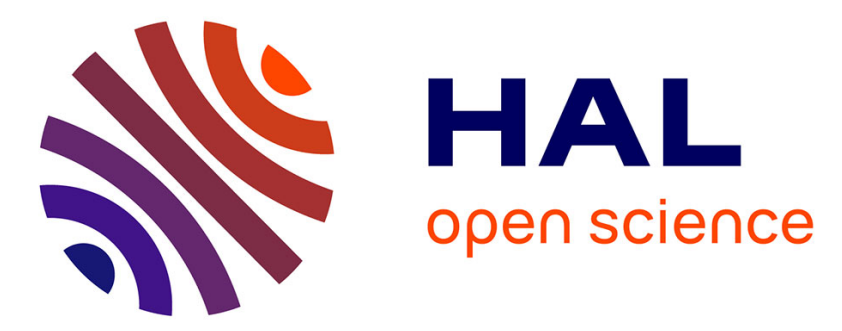

\title{
Rear-side resonator architecture for the passive coherent combining of high-brightness laser diodes
}

\author{
Guillaume Schimmel, Ioana Doyen-Moldovan, Sylvie Janicot, Marc Hanna, \\ Jonathan Decker, Paul Crump, G Blume, Götz Erbert, Patrick Georges, \\ Gaëlle Lucas-Leclin
}

\section{To cite this version:}

Guillaume Schimmel, Ioana Doyen-Moldovan, Sylvie Janicot, Marc Hanna, Jonathan Decker, et al.. Rear-side resonator architecture for the passive coherent combining of high-brightness laser diodes. Optics Letters, 2016, 41 (5), pp.950-953. 10.1364/OL.41.000950 . hal-01282939

\section{HAL Id: hal-01282939 \\ https://hal-iogs.archives-ouvertes.fr/hal-01282939}

Submitted on 11 Mar 2016

HAL is a multi-disciplinary open access archive for the deposit and dissemination of scientific research documents, whether they are published or not. The documents may come from teaching and research institutions in France or abroad, or from public or private research centers.
L'archive ouverte pluridisciplinaire HAL, est destinée au dépôt et à la diffusion de documents scientifiques de niveau recherche, publiés ou non, émanant des établissements d'enseignement et de recherche français ou étrangers, des laboratoires publics ou privés.

\section{(1) $(1) \Theta$}

Distributed under a Creative Commons Attribution - NonCommercial - NoDerivatives| 4.0 


\title{
Rear-side resonator architecture for the passive coherent combining of high-brightness laser diodes
}

\author{
G. SCHIMmel ${ }^{1}$, I. DOYen-Moldovan ${ }^{1}$, S. JANICOT ${ }^{1}$, M. HANNA ${ }^{1}$, J. DeCKeR ${ }^{2}$, \\ P. CRUMP ${ }^{2}$, G. BLUME ${ }^{2}$, G. ERBERT ${ }^{2}$, P. GEORGES ${ }^{1}$, G. LUCAS-LECLIN ${ }^{1 *}$ \\ ${ }^{1}$ Laboratoire Charles Fabry, Institut d'Optique Graduate School, CNRS, Université Paris-Saclay, 91127 Palaiseau cedex, France \\ ${ }^{2}$ Ferdinand-Braun-Institut, Leibniz-Institut für Höchstfrequenztechnik, Gustav-Kirchhoff-Straße 4, 12489 Berlin, Germany \\ *Corresponding author: gaelle.lucas-leclin@institutoptique.fr
}

Received 1 December 2015; revised 26 January 2016; accepted 26 January 2016; posted 27 January 2016 (Doc. ID 254861); published 22 February 2016

\begin{abstract}
We describe a new coherent beam combining architecture based on passive phase-locking of emitters in an extended cavity on their rear facet, and their coherent combination on the front facet. This rear-side technique provides strong optical feedback for phaselocking while maintaining a high electrical-to-optical efficiency. Two high-brightness high-power tapered laser diodes are coherently combined using a Michelson-based cavity - the combining efficiency is above $82 \%$ and results in an output power of $6.7 \mathrm{~W}$ in a nearly diffraction-limited beam with an $M^{2}{ }_{4 \sigma} \leq 1.2$. A semi-active automatic adjustment of the current enhances the long-term stability of the combination, while the short-term stability is passively ensured by the extended cavity. This new laser configuration exhibits the simplicity of passive self-organizing architectures, while providing a power conversion efficiency of $27 \%$ that is comparable to masteroscillator power amplifier architectures.
\end{abstract}

OCIS codes: (140.2020) Diode lasers; (140.3298) Laser beam combining; (140.5960) Semiconductor lasers.

http://dx.doi.org/10.1364/OL.41.000950

Scaling up the brightness of laser diodes has been a major re- search objective in the laser community in the past few decades. It is now generally accepted that the power density of single- mode diode lasers and, consequently, their radiance, is ultimately limited by catastrophic optical damage (COD)-induced device failure [1]. The most efficient technique to overcome this limit and increase the brightness regardless of laser type is beam combination of several emitters. While spectral beam combining techniques use laser emitters at different wave- lengths, coherent beam combining (CBC) consists of the superposition of individual laser beams by constructive interference, providing a single high-power laser beam with excellent spectral and spatial properties [2]. For CBC to be effective, individual laser elements must have a proper phase relationship that

remains constant over time, which can only be achieved in an arrangement that forces the required phase relation between the emitters. In all practical CBC implementations, laser beams with identical and single-transverse mode profiles are combined. Over the years, different approaches were investigated: either active phase locking of amplifiers seeded by a single-frequency laser split into $\mathrm{N}$ beams and amplified in par- allel in the individual amplifiers, or passive self-organization of emitters in a common laser cavity [3]. The feasibility of such architectures was brilliantly demonstrated with fiber and solid state lasers, showing impressive performance in the past few years [4]. With semiconductor lasers and amplifiers, the highest output power and the largest number of phase-locked semiconductor amplifiers (respectively, $40 \mathrm{~W}$ and 218) were demonstrated in the master oscillator power amplifier (MOPA) configuration by Creedon et al. at MIT Lincoln Laboratory [5,6]. The MOPA architecture leads intrinsically to a high electrical-to-optical (E-0) conversion efficiency, but requires non-standard devices and real-time active correction of the phase. On the other hand, different extended cavity setups are described in the literature, based on self-imaging [7-9] and interferometric architectures [10-12] to force passive phase locking of lasers. Nevertheless, with such passive cavities, the coherence of the laser array drops as the operating current increases and the need for a strong optical feedback on the front facet of the lasers reduces their E-O conversion efficiency. Active correction of the lasers was demonstrated to allow phase-locked operation at high currents, but still with a low extracted power [10]. Efforts to improve the E-O efficiency of passively phase-locked arrays were also described in [13] in a V-shaped external cavity designed for broad area laser diodes, pointing out the importance of the cavity architecture to get both high power and efficiency.

In the following, we investigate a new $\mathrm{CBC}$ architecture using a common extended cavity on the back side of diode lasers for phase locking, while the coherent beam superposition of the phase-locked beams is realized on the front side. As a result, the E-O conversion efficiency of the phase-locked laser cavity is increased as compared to standard front-side configurations. Moreover, such an extended cavity 
placed on the rear side provides the strong optical feedback required for phase-locked operation. This configuration is demonstrated with two high- brightness tapered devices [14] in a Michelson-type extended cavity $[15,16]$, highlighting the capability of such a setup for high power operation.

The tapered laser devices used are emitting around $\lambda=976 \mathrm{~nm}$. The lasers contain a $2 \mathrm{~mm}$ long ridge section, and a $4 \mathrm{~mm}$ long tapered section ( $\alpha_{\mathrm{T}}=6^{\circ}$ taper angle). The front (tapered section) facet has a $0.5 \%$ reflectivity, while the rear (ridge section) facet has a $\mathrm{R}<0.1 \%$ coating. Details of the design of the tapered diodes are given in [17]. The tapered lasers are mounted p-side up on C-Mount to allow access to both facets. The two sections are separately driven by currents $I_{R}$ and $\mathrm{I}_{\mathrm{T}}$, respectively. With a high-reflectivity extended cavity on their rear facets, the extracted optical power reaches $4 \mathrm{~W}$ at $\mathrm{I}_{\mathrm{R}}=400 \mathrm{~mA}$ and IT $6 \mathrm{~A}$, corresponding to an E-O con- version efficiency of 33\%. The beam is diffraction limited along the fast axis; along the slow axis, the beam quality factor is $\mathrm{M}^{2} 4 \sigma \approx 2.5$ at $\mathrm{I}_{\mathrm{T}} 6 \mathrm{~A}$ with about $80 \%$ of the extracted power contained in the diffraction-limited central lobe. The radiance is $\mathrm{B}=\mathrm{P} / \lambda^{2} \times \mathrm{M}^{2} \approx 200 \mathrm{MW} \cdot \mathrm{cm}^{-2} \cdot \mathrm{sr}^{-1}$ at $6 \mathrm{~A}$. For such lasers, separate (rear side) phase locking and (front side) coherent combining is the preferred and most promising configuration, as optical feedback into the front facet (tapered section) might deteriorate the beam quality and even lead to early device failure, and as the phase locking is obtained with diffraction limited beams from the ridge sections.

We now describe the phase-locking architecture with two emitters. The extended cavity is based on a Michelson interferometer on the rear side featuring an external reflector. The front facets of each diode are the output couplers of the laser cavity, coated to yield $0.5 \%$ reflectivity which is sufficient to achieve lasing owing to the high gain in the diode medium. The cavity backend features an external 2000 lines/mm diffraction grating (at Littrow incidence), which serves as a common, dispersive high reflector and controls the wavelength (see Fig. 1). This ensures a stable and narrow laser line at $976 \mathrm{~nm}$. Both laser beams are collimated with high NA aspheric lenses $\left(\mathrm{F}_{1} \& \mathrm{~F}_{2}\right)$ and combined on a $50 / 50$ beam splitter $\left(B_{1}\right)$. Since both lasers share the same extended cavity, they undergo minimum losses if the beams are in phase at $\mathrm{BS}_{1}$, resulting in constructive interference on the $\mathrm{P}$ arm and destructive interference on the other arm. On the other hand, incoherent operation of both lasers induces 75\% losses per roundtrip in the cavity for each laser. Coherent operation, thus, is strongly favored by the Michelson extended cavity.

On the taper (front) side, an arrangement of an aspheric lens and a cylindrical lens $\left(\mathrm{F}_{1}^{\prime} \& \mathrm{~F}_{2}^{\prime}\right)$ is used to correct for the intrinsic astigmatism of the tapered laser devices, and a simple 50/50 beam splitter $\left(\mathrm{BS}_{2}\right)$ is used as a combiner to perform coherent superposition of the beams. A phase plate $\mathrm{L}_{\Phi}$ (an anti-reflection coated $0.5 \mathrm{~mm}$ thick plane silica plate) is added on one arm to adjust the phase relationship between the two laser beams, as their optical paths are different. Rotating the plate allows fine tuning of the phase difference and maximizing of the combined power in the $\mathrm{P}_{0} \mathrm{arm}$.

The extended cavity (which is formed from the common backend external grating to each emitter front facet) performs the phaselocking function. From BS1 to the rear facets of each emitter, the arms are 25 and $160 \mathrm{~mm}$ long, respectively. The passive phase-locking operation of the two lasers is realized for currents up to $\mathrm{I}_{\mathrm{T}}=6 \mathrm{~A}$ (five times the laser threshold). On the front side, the laser power extracted from each device is $\mathrm{P}_{1}=\mathrm{P}_{2} \approx 4 \mathrm{~W}$ at $\mathrm{I}_{\mathrm{R}}=400 \mathrm{~mA}$ and $\mathrm{I}_{\mathrm{T}}=6 \mathrm{~A}$ in phaselocked operation. The maximum combined optical power $\mathrm{P}_{0}$ (see Fig. 1) is $6.7 \mathrm{~W}$, which corresponds to a net efficiency, defined as $\eta \mathrm{P}^{\prime}=\mathrm{P}^{\prime} /\left(\mathrm{P}_{1}\right.$ $+\mathrm{P}_{2}$ ), in excess of $82 \%$. By this definition, this figure also accounts for all loss mechanisms inherent in our system. Experimentally, we observe that the extended cavity acts as a lateral mode filter: the slow axis (SA) beam quality for each emitter is improved to $\mathrm{M}^{2} 4 \sigma \leq 1.3$ when both emitters are phase-locked at $\mathrm{I}_{\mathrm{T}}=6 \mathrm{~A}$ [seeFig.2(b)]. Since the combining stage on the front facets operates as a second spatial filter, the beam quality of the combined beam is further enhanced to $\mathrm{M}^{2} 4 \sigma \leq 1.2$ [see Fig. 2(d)]. Indeed, only the common features in the spatial intensity and phase of both beams are coherently combined. This results in a spatial cleaning of the combined beam, whereby lateral modes are rejected on the loss arm. Thus, the radiance of the laser system is enhanced to $\mathrm{B} \approx$ $500 \mathrm{MW} \cdot \mathrm{cm}^{-2} \cdot \mathrm{sr}^{-1}$, with $6.7 \mathrm{~W}$ of combined power. The corresponding E-O conversion efficiency is $27 \%$, higher than what is achieved in standard front-side extended cavities [8-10] owing to the low reflectivity coatings on the front facets, which is made possible by our rear-side architecture.

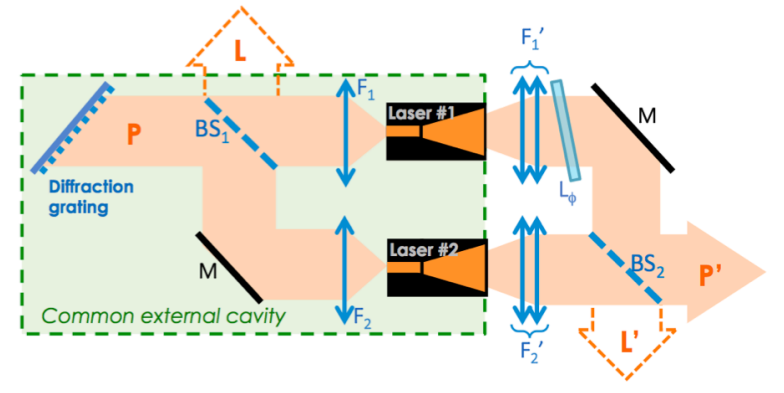

Fig. 1. Experimental set-up with the rear-side Michelson extended cavity and the front-side coherent superposition; $\mathrm{BS}_{1-2: 50}$ 50/50 beamsplitters; P, P': useful arms; L, L': losses. ; F1,2: 8 mm aspheric lenses; $F_{1}^{\prime}, 2$ : combination of $2.75 \mathrm{~mm}$ aspheric lenses and $19 \mathrm{~mm}$ cylindrical lenses; M: high reflective mirrors.

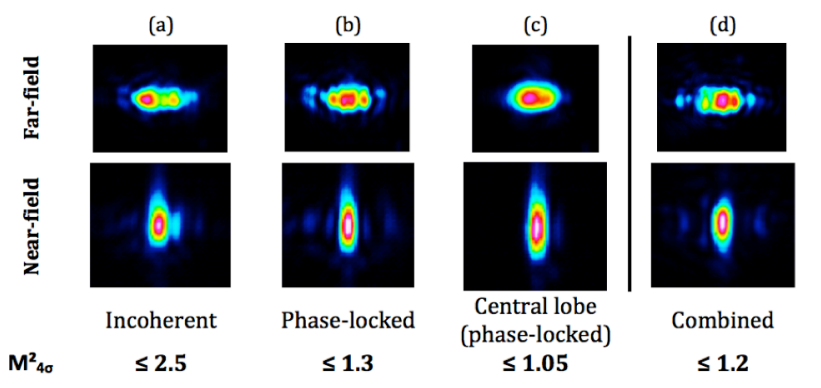

Fig. 2. Intensity profiles of the far field (top) and near field (bottom) for laser 1 alone (a, b, c) and the combined output (d). Vertical and horizontal directions correspond respectively to the fast and slow-axes. The $\mathrm{M}^{2}$ values are given for the slow-axis. 
Table 1. Combining results for the full beams and for the central lobes $-I_{R}$ is set to $400 \mathrm{~mA}$

\begin{tabular}{c|c|ccc|ccc}
\hline & Individual laser & \multicolumn{3}{|c|}{ Whole beam - w/o SA spatial filtering stage } & \multicolumn{3}{|c}{ Central lobe - with SA spatial filtering stage } \\
\hline $\mathbf{I}_{\mathbf{T}}(\mathbf{A})$ & Output power $\mathrm{P}_{1}(\mathrm{~W})$ & $\mathrm{P}_{1}+\mathrm{P}_{2}(\mathrm{~W})$ & Comb. power $\mathrm{P}^{\prime}(\mathrm{W})$ & $\eta^{\prime}$ & $\mathrm{P}_{1}+\mathrm{P}_{2}(\mathrm{~W})$ & Comb. power $\mathrm{P}^{\prime}(\mathrm{W})$ & $\eta^{\prime}$ \\
\hline $\mathbf{3}$ & 1.5 & 2.9 & 2.5 & $85 \%$ & 2.4 & 2.2 & $95 \%$ \\
\hline $\mathbf{4 . 5}$ & 2.8 & 5.6 & 4.7 & $85 \%$ & 4.4 & 4.1 & $63 \%$ \\
\hline $\mathbf{6}$ & 4 & 8.1 & 6.7 & $82 \%$ & 6.5 & $62 \%$ \\
\hline
\end{tabular}

The combining efficiency is limited by several factors, such as the proportion of incoherent light or mismatches between the beams [18]. To illustrate the high coherence between beams to be combined, a two-wave interference pattern is obtained by crossing the beams at a large angle [19]. In the central part of the beams, dominated by the fundamental mode content, the visibility is $96 \%$ and can be shown to be a lower bound of the mutual coherence [20], indicating that the ASE is not a limitation (confirmed with a spectrum analysis). The remaining origins of beam combining efficiency decrease come from mismatches in intensity and phase profiles. Since the beams are nearly single transverse mode, these mismatches can be separated into two parts: differences in higher-order mode content and differences in fundamental mode profiles. To identify these contributions, first the intensity mismatches for the full beams are quantified from the overlap calculation between both measured intensity profiles [21] leading to $9 \%$ reduction of the combining efficiency at $\mathrm{I}_{\mathrm{T}}=6 \mathrm{~A}$. Second, we isolate the fundamental mode content by implementing a spatial filtering stage on the combined arm $\mathrm{P}_{0}$ for the slow axis (SA). The central lobe of the beams is selected, corresponding to $80 \%$ of the optical output power for each beam [Fig. 2(c)]. The combined optical power in this case is $6 \mathrm{~W}$, indicating that the combining efficiency is significantly improved from $82 \%$ to $92 \%$ at $\mathrm{I}_{\mathrm{T}}=6 \mathrm{~A}$ and up to $95 \%$ at $3 \mathrm{~A}$. These results are summarized in Table 1 . A 5\% decrease in efficiency in this case can be identified as a consequence of a residual astigmatism difference between fundamental modes. With SA filtering, the radiance reaches $600 \mathrm{MW} \cdot \mathrm{cm}^{-2} \cdot \mathrm{sr}^{-1}$. The filtered output power of $6 \mathrm{~W}$ demonstrates that $89 \%$ of the combined power extracted directly from the laser system before the filtering stage is into the diffraction-limited central lobe.

In our standard laboratory environmental conditions, we observe that the front output power remains stable, typically for several minutes, before the phase relationship between both laser beams on the front side jumps to a different phase state. These phase jumps are the result of mode-hopping of the laser line from one longitudinal mode of the extended cavity to an- other, arising from external perturbations [22]. As the optical path lengths from BS1 to BS2 are different for the two laser beams, a change in the wavelength leads to a phase shift on BS2, causing a drop of the combining efficiency. Equalizing on the millimeter scale the optical path lengths could minimize it. Figure 3 illustrates the effect of mode hopping with a measurement of the front phase relationship between the beams at BS2 as a function of time, while several phase shifts occur. The output power on the rear side loss arm $L$ and the wavelength are measured simultaneously. The amplitude of the mode-hop is of a few freespectral ranges of the interferometric cavity (FSR $\approx 3 \mathrm{pm}$ ). Two different front phase-states are observed while the phase-locked operation is maintained-indeed, the back optical losses remain significantly below the incoherent level (represented by a red line on Fig. 3, bottom), meaning that destructive interference occurs on arm $\mathrm{L}$. At $\mathrm{t}>16 \mathrm{~s}$, we also observe a competition between coherent and incoherent operations of the cavity, characterized by high extendedcavity losses on arm $\mathrm{L}$, unstable lasing wavelength and undefined front phase relationship. On the other hand, between two jumps of the operating point, a single phase-state is passively maintained with residual phase fluctuations estimated to 20 mrad RMS.

To get a better understanding of the passive stability provided by the cavity during a stable phase-state operation, a known phase perturbation is intentionally applied on one emit- ter to observe the laser system behavior. The perturbation is a modulation of the ridge current of $\delta \mathrm{I}_{\mathrm{R}}=0.4 \mathrm{~mA}$ amplitude at $1.2 \mathrm{kHz}$, resulting in a modulation $\delta \Delta \mathrm{L}$ of the optical path length of one arm only of the interferometric cavity and corresponding to a single-pass phase-shift $\delta \varphi$ of about $\pi 90$. The modulation is small enough to prevent any mode hopping. As a result, we observe a simultaneous modulation of the lasing frequency, measured with a Fabry-Perot interferometer. The frequency modulation amplitude is $\delta v=10 \mathrm{MHz}$, corresponding to a relative frequency shift $\delta \mathrm{w}$ compensating for the relative optical path-length perturbation $\delta \Delta \mathrm{L} / \Delta \mathrm{L}$, with $\Delta \mathrm{L}$ the optical path-length difference between the two arms from the front laser facets to BS1. This has the effect of maintaining the relative phase difference $\Delta \varphi$ between the beams on BS1, $\Delta \varphi 2 \pi / \mathrm{c} \times \nu \times \Delta \mathrm{L}$. Thus, we can conclude that the passive phase-locking operation of the lasers is maintained through two effects: the longitudinal mode of the cavity is continuously selfadapting to small and short-term perturbations on the MHz-scale, while mode-hops of several FSR in the GHz-range ensure the long-term stability, compensating for the strongest perturbations.

Even though the phase-locked operation of the cavity is stable, the combining efficiency is no longer maximum after a longitudinal modehop because of the front-side phase change on BS2. Thus the orientation of the phase plate $\mathrm{L} \varphi$ has to be corrected after each brutal phase shift to maximize the combined power on the $\mathrm{P}^{\prime}$ arm in the longterm. Here we propose to automatically maintain the coherent combining efficiency $\eta^{\prime}$ to its maximum value, with a semi-active correction of the driving currents of the lasers-self activated when necessary only. The electronic feedback loop assists the combination of the beams compensating for the drop of the efficiency caused by mode hopping and the phase locking of the lasers correcting for long-term perturbations. The power L' on the front side is measured at a $10 \mathrm{~Hz}$ rate, and the currents are automatically changed to minimize it. The minimization algorithm uses an iterative random exploration of the currents around their nominal values, with a $0.5 \mathrm{~mA}$ maximum amplitude at each step. Due to the highly nonlinear behavior of the laser cavity, it is noteworthy that the currents are not optimized; strictly speaking, they are corrected to ensure the proper phase relationship on the front side, while the extended cavity self-adjusts to maintain phase locking. The active correction runs if only the power on arm L' increases above a fixed threshold. This long-term electronic correction of the operating currents completes the passive optical feedback by the extended cavity on the rear side and stabilizes the coherent beam combining efficiency $\eta$ ', which remains higher than $75 \%$ (compared to the maximum 82\%) in a 15 min average (see Fig. 4). The abrupt jumps of the optical power correspond to wrong solutions at the start of the random exploration.

In conclusion, we demonstrated a CBC concept for diode lasers, in which the emitters are passively phase locked by means of an extended rear-side cavity. In this Letter, we imple-mented our concept with two tapered lasers in a Michelson- type cavity configuration and 


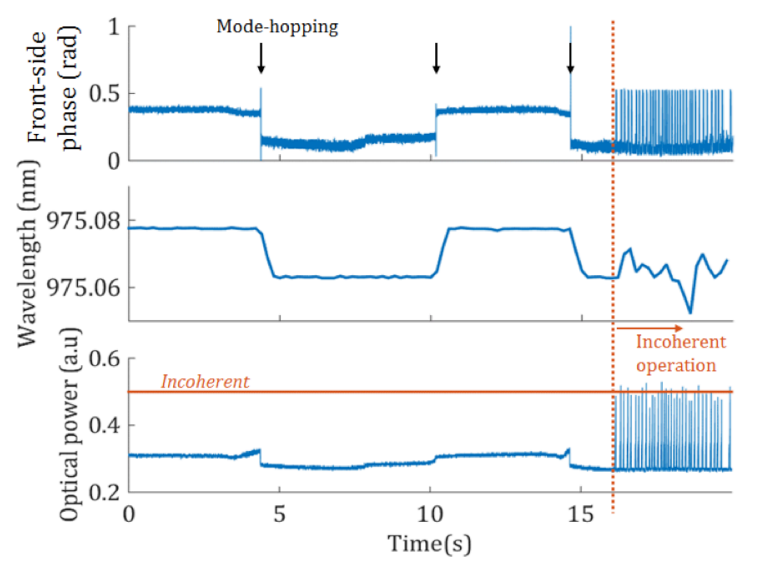

Fig. 3. Relative front-phase between the two beams on $\mathrm{BS}_{2}$ (top), wavelength (middle) and back optical losses on arm L (bottom) function of time. The wavelength is measured with a $1 \mathrm{pm}$ resolution wave-meter. The system passively maintains coherent operation for about 16 seconds, after which the accumulated phase fluctuations become excessive.

used a semi-active feedback control of the diode drive currents to retain long-term coherently phased operation. An optical power of $6.7 \mathrm{~W}$ has been extracted from the laser system with $>82 \%$ net combining efficiency. Our architectures provide minimal coherence degradation at high drive current, and its benefits are enhanced by our use of tapered devices which ensures high brightness per emitter, although our concept is suitable for different laser designs [19,23].

To the best of our knowledge, the optical power reported in this Letter is the highest achieved in a single beam by coherently combined diode lasers using a self-organized extended cavity.

The high E-O conversion efficiency and algorithmic simplicity/low bandwidth of our control feedback loop makes our concept especially attractive for scaling to $\mathrm{N}$ emitters, which can be pursued, for example, by means of different interferometric [10-12] or self-imaging cavity [7-9] designs.

Funding. European Union $7^{\text {th }}$ Framework Program (314719).

Acknowledgment. We thank Katrin Paschke from FBH for detailed technical discussions.

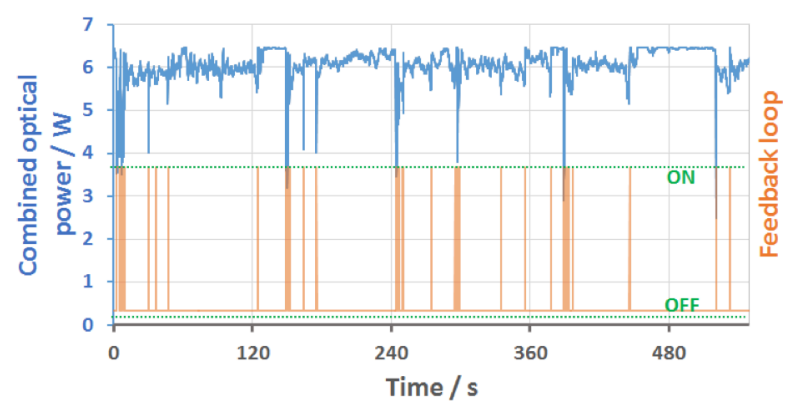

Fig. 4. Combined optical power on arm P' (in blue) and state of the electronic feedback loop (in orange) as a function of time. The upstate of the orange curve corresponds to a closed loop (ON) while the down-state (OFF) corresponds to an open loop. The average optical power is $6.05 \mathrm{~W}$ with a RMS value of $0.4 \mathrm{~W}$.

\section{References}

1. J. W. Tomm, M. Ziegler, M. Hempel, and T. Elsaesser, Laser Photonics Rev. 5, 422-441 (2011).

2. T. Y. Fan, IEEE J. Sel. Top. Quantum Electron. 11, 567-577 (2005).

3. A. Brignon, (John Wiley \& Sons, 2013).

4. S. M. Redmond, D. J. Ripin, C. X. Yu, S. J. Augst, T. Y. Fan, P. A. Thielen, J. E. Rothenberg, and G. D. Goodno, Opt. Lett. 37, 2832 (2012).

5. S. M. Redmond, K. J. Creedon, J. E. Kansky, S. J. Augst, L. J. Missaggia, M. K. Connors, R. K. Huang, B. Chann, T. Y. Fan, G. W. Turner, and A. SanchezRubio, Opt. Lett. 36, 999 (2011).

6. K. J. Creedon, S. M. Redmond, G. M. Smith, L. J. Missaggia, M. K. Connors, J. E. Kansky, T. Y. Fan, G. W. Turner, and A. Sanchez-Rubio, Opt. Lett. 37, 5006 (2012).

7. R. K. Huang, B. Chann, L. J. Missaggia, S. J. Augst, M. K. Connors, G. W. Turner, A. Sanchez-Rubio, J. P. Donnelly, J. L. Hostetler, C. Miester, and F. Dorsch, Proc. SPIE 7230, 72301G (2009).

8. D. Paboeuf, G. Lucas-Leclin, P. Georges, N. Michel, M. Krakowski, J. Lim, S. Sujecki, and E. Larkins, Appl. Phys. Lett. 93, 211102 (2008).

9. C. J. Corcoran and F. Durville, Opt. Express 22, 8420 (2014).

10. J. Montoya, S. J. Augst, K. Creedon, J. Kansky, T. Y. Fan, and A. SanchezRubio, Appl. Opt. 51, 1724 (2012).

11. G. Bloom, C. Larat, E. Lallier, G. Lehoucq, S. Bansropun, M.-S. L. LeeBouhours, B. Loiseaux, M. Carras, X. Marcadet, G. Lucas-Leclin, and P. Georges, Opt. Lett. 36, 3810 (2011).

12. J. R. Leger, G. J. Swanson, and W. B. Veldkamp, Appl. Opt. 26, 4391 (1987).

13. B. Liu and Y. Braiman, Opt. Express 21, 31218 (2013).

14. H. Wenzel, B. Sumpf, and G. Erbert, Comptes Rendus Phys. 4, (2003).

15. G. Bloom, C. Larat, E. Lallier, M. Carras, and X. Marcadet, Opt. Lett. 35, 1917 (2010).

16. D. Sabourdy, V. Kermène, A. Desfarges-Berthelemot, M. Vampouille, and A. Barthélémy, Appl. Phys. B 75, 503-507 (2002).

17. C. Fiebig, G. Blume, C. Kaspari, D. Feise, J. Fricke, M. Matalla, W. John, H. Wenzel, K. Paschke, and G. Erbert, Electron. Lett. 44, 1253-1255 (2008).

18. G. D. Goodno, C.-C. Shih, and J. E. Rothenberg, Opt. Express 18, 25403 (2010).

19. G. Schimmel, I. Doyen, S. Janicot, L. P. Ramirez, M. Hanna, P. Georges, G. Lucas-Leclin, V. Vilokkinen, P. Melanen, P. Uusimaa, J. Decker, P. Crump, G. Erbert, S. Bull, S. Kaunga-Nyirenda, and E. C. Larkins, Proc. SPIE 9348, 93480P (2015).

20. L. Mandel and E. Wolf, (Cambridge University Press, 1995).

21. P. A. Thielen, J. G. Ho, D. A. Burchman, G. D. Goodno, J. E. Rothenberg, M. G. Wickham, A. Flores, C. A. Lu, B. Pulford, C. Robin, A. D. Sanchez, D. Hult, and K. B. Rowland, Opt. Lett. 37, 3741 (2012).

22. D. Sabourdy, V. Kermene, A. Desfarges-Berthelemot, L. Lefort, A. Barthelemy, P. Even, and D. Pureur, Opt. Express 11, 87 (2003).

23. H. Bruesselbach, D. C. Jones, M. S. Mangir, M. Minden, and J. L. Rogers, Opt. Lett. 30, 1339 (2005). 